\title{
Sudden death of COVID-19 patients in Wuhan, China: A retrospective cohort study
}

\author{
Nan Yang ${ }^{1 *}$, Kunming Tian ${ }^{3 *}$, Meng Jin ${ }^{4 *}$, Xu Zhang ${ }^{5 *}$, \\ Fengqin Zhang ${ }^{2 *}$, Xiuquan $\mathrm{Shi}^{8}$, Xiaoyang Wang ${ }^{1}$, Si- \\ yuan $\mathrm{Niu}^{1}$, Jing $\mathrm{Shi}^{2}$, Ke $\mathrm{Hu}^{4}$, Kui Liu${ }^{2}$, Ping Peng7${ }^{7}$, Ying \\ Wang ${ }^{6}$, Huilan Zhang ${ }^{2}$, Jianbo Tian ${ }^{1}$ \\ ${ }^{1}$ Department of Epidemiology and Biostatistics, Key Laboratory for \\ Environment and Health, School of Public Health, Tongji Medical College, \\ Huazhong University of Sciences and Technology, Wuhan, China \\ ${ }^{2}$ Department of Respiratory and Critical Care Medicine Tongji Hospital, \\ Tongji Medical College, Huazhong University of Science and Technology, \\ Wuhan, China \\ ${ }^{3}$ Institute of Reproductive Health, Tongji Medical College, Huazhong \\ University of Science and Technology, Wuhan, China \\ ${ }^{4}$ Department of Respiratory and Critical Care Medicine, Renmin Hospital of \\ Wuhan University, Wuhan, China \\ ${ }^{5}$ Key Laboratory of Environment and Health, Ministry of Education \& Ministry \\ of Environmental Protection, and State Key Laboratory of Environmental \\ Health (Incubating), School of Public Health, Tongji Medical College, \\ Huazhong University of Science and Technology, Wuhan, China \\ ${ }^{6}$ Department of Virology, Wuhan Centers for Disease Prevention and Control, \\ Wuhan, China \\ ${ }^{7}$ Department of Oncology, Tongji Hospital, Tongji Medical College, \\ Huazhong University of Science and Technology, Wuhan, China \\ ${ }^{8}$ Department of Epidemiological and health statistics, School of Public \\ Health, Zunyi Medical University, Zunyi, China \\ * Equal first authorship.
}

\section{Correspondence to:}

\section{Jianbo Tian}

Department of Epidemiology and Biostatistics

Key Laboratory for Environment and Health

School of Public Health

Tongji Medical College

Huazhong University of Sciences and Technology

Wuhan 430030, China

tianjianbo1992@126.com

Ping Peng

Department of Oncology

Tongji Hospital

Tongji Medical College

Huazhong University of Science and Technology

Wuhan 430030, China

pengpingtj@163.com
Background In December 2019, coronavirus disease 2019 (COVID-19) emerged in Wuhan city and rapidly spread throughout China. So far, it has caused 4000 deaths in this country. We aimed to systematically characterize clinical features and determine risk factors of sudden death for COVID-19 patients.

Methods: Deceased patients with COVID-19 in Tongji Hospital from January 22 to March 23, 2020 were extracted. Patients who died within 24 hours after admission were identified as sudden deaths, and the others formed non-sudden deaths. The differences in clinical characteristics between the two groups were estimated. Risk factors associated with sudden deaths were explored by logistic regression.

Results 281 deceased patients were enrolled in this study. Sudden death occurred in $28(10.0 \%)$ patients, including 4 (14.3\%) admitted to the intensive care unit. Fatigue was more common in sudden deaths $(11,47.8 \%)$ than in non-sudden deaths (40, $17.2 \%)$. Both the count and percentage of eosinophils were lower in sudden deaths than that in non-sudden deaths $(\mathrm{P}=0.006$ and $\mathrm{P}=0.004)$. Compared with non-sudden deaths, sudden deaths had higher plasma levels of procalcitonin, C-reactive protein, D-dimer, alanine aminotransferase, aspartate aminotransferase, gamma-glutamyl transferase, lactate dehydrogenase, alkaline phosphatase and N-terminal pro-brain natriuretic peptide. There were not significant differences in gender, age, chest CT image features and comorbidities observed.

Conclusions The differences between the two groups suggested more severe systemic inflammation, multi-organ dysfunction, especially impaired liver and heart function in COVID-19 patients who died suddenly after admission. More researches are needed to verify these points. 
In December 2019, a series of pneumonia cases of unknown cause were identified in Wuhan, China [1]. This pneumonia, later named as coronavirus disease 2019 (COVID-19) by WHO, was caused by a novel enveloped RNA betacoronavirus, SARS-CoV-2 [2]. Owing to the high infectivity and pathogenicity of the virus, COVID-19 has rapidly spread worldwide $[3,4]$.

Case fatality rate was assessed as high as $1 \%$, which is much greater than seasonal influenza at about $0.1 \%$ [5]. In China, the case fatality rate was $17.3 \%$ in the early stages of the outbreak and reduced over time to $0.7 \%$ after 1 February [6]. With the improvement of monitoring measures and medical treatment methods, the survival rate of patients has been greatly improved. However, there were still a small number of patients died within a short period of time after admission, in other words, sudden death. As no vaccine or specific antiviral therapy against COVID-19 has been proven to be effective, it is beneficial to implement supportive therapy to relieve symptoms and protect multiple organ functions. Treating patients at risk of sudden death promptly is essential to reduce mortality.

In this study, we collected data of deceased patients with COVID-19 in Tongji Hospital (Wuhan, China). By comprehensively evaluating the demographic, clinical, radiological, and laboratory characteristics between patients who died within 24 hours after admission and those who died over 24 hours after admission, we hope to find valuable markers related to sudden death.

\section{METHODS}

\section{Patients}

This single-center, retrospective study was performed at Tongji Hospital (Wuhan, China), which is a designated hospital to treat patients with COVID-19. All patients in this study were diagnosed with COVID-19 on the basis of World Health Organization interim guidance [7], and died between January 22 and March 23, 2020. patients who died within 24 hours after admission were defined as sudden deaths, while those who died more than 24 hours after admission were defined as non-sudden deaths. In total, 281 deceased patients with COVID-19 were included, consisting of 28 sudden deaths and 253 non-sudden deaths. This study was approved by the Ethics Committee of Tongji Hospital, Tongji Medical College, Huazhong University of Science and Technology.

\section{Laboratory procedures}

Methods for laboratory confirmation of SARS-CoV-2 infection are standardized. Pharyngeal swabs were collected from patients and placed into tubes with virus preservation solution. Total RNA was extracted within two hours using respiratory sample RNA isolation kits (Pfizer or BioGerm, Shanghai, China). Two target genes, open reading frame $\mathrm{lab}(\mathrm{ORF} \mathrm{lab})$ and nucleocapsid protein $(\mathrm{N})$, were simultaneously amplified and tested by real time RT-PCR assay, which was conducted using SARS-Cov-2 nucleic acid detection kits (BioGerm, Shanghai, China) according to the manufacturer's protocol. The reaction mixture contained $12 \mu \mathrm{L}$ of reaction buffer, $4 \mu \mathrm{L}$ of enzyme solution, $4 \mu \mathrm{L}$ of ORFlab/N primers solution, $3 \mu \mathrm{L}$ of RNase-Free Water, and $2 \mu \mathrm{L}$ of RNA template. The RT-PCR reaction was under the following conditions: incubation at $50^{\circ} \mathrm{C}$ for 15 minutes and $95^{\circ} \mathrm{C}$ for 5 minutes, and 40 cycles of denaturation at $95^{\circ} \mathrm{C}$ for 10 seconds and fluorescence signal acquisition at $55^{\circ} \mathrm{C}$ for 45 seconds. Results were defined as negative (cycle threshold value $>38$ or not detected), positive (amplification curve was s-shaped, and cycle threshold value $\leq 35$ ) and suspicious (amplification curve was s-shaped, and $35<$ cycle threshold value $\leq 38$ ). In addition, nucleic acid test positive interpretation criteria are divided into two aspects: first, in the same specimen, ORFlab and N genes were tested positive at the same time; second, the ORFlab or N gene was positive in two different samples of the same patient. These criteria are based on the recommendation by the National Institute for Viral Disease Control and Prevention (China).

Other laboratory tests included complete blood count, serum biochemical tests (including liver and kidney function, creatine kinase, lactate dehydrogenase, and electrolytes), coagulation profile and cytokine tests. Chest CT scan were also done for inpatients. Frequency of examinations was determined by the physicians.

\section{Data collection}

Demographic, clinical, radiological and laboratory characteristics and treatment data were collected from electronic medical records. If a patient received a same examination more than once, we only extracted the first result of the examination. All data were reviewed independently by two researchers. 


\section{Statistical analysis}

Categorical variables were presented as numbers and percentages. Continuous variables were presented as mean and standard deviation (SD) if they were normally distributed, or median and interquartile range (IQR) if they were not. Means for continuous variables were compared using independent sample $t$ tests. Medians for continuous variables were compared using Wilcoxon rank sum tests. Categorical variables were compared using $\chi^{2}$ or Fisher exact tests. Univariable and multivariable logistic regression models were employed to explore risk factors associated with sudden death of COVID-19 patients. In multivariable logistic regression models, factors including age, sex and each comorbidity were adjusted. All tests were two sided. $P$ values less than 0.05 were defined as statistically significant. IBM SPSS Statistics 22 (IBM Corp, Armonk NY, USA) was employed for all analyses.

\section{RESULTS}

\section{Demographic features, clinical comorbidities and symptoms}

The median age of all patients was 69.0 (IQR=62.0-77.0). The ratio of male to female was 2.1. Most patients had comorbidities, of which hypertension was the most common type (38.8\%), followed by diabetes (14.2\%) and coronary heart disease (11.4\%). There were not significant differences in age, gender and clinical comorbidities between sudden and non-sudden deaths. Fever was the most prevalent symptom in both sudden deaths $(18,78.3 \%)$ and non-sudden deaths $(177,76.0 \%)$, but the proportions of patients in the two groups were not comparable. Besides, for analyses on symptoms including dyspnea, diarrhea, chest tightness, chills, poor appetite, muscle pain, headache and vertigo, no significant differences were detected. Cough and expectoration were more common in non-sudden deaths $(154,66.1 \%$ and $102,43.8 \%)$ than in sudden deaths (7, $30.4 \%$, and $4,17.4 \%)$. Fatigue was much more common in sudden deaths $(11,47.8 \%)$ than in non-sudden deaths $(40,17.2 \%)$. Results are shown in Table 1.

Table 1. Clinical characteristics, radiographic findings and treatments of the study patients*

\begin{tabular}{|c|c|c|c|c|}
\hline INDICATORS & TOTAL & NON-SUDDEN DEATHS & SUDDEN DEATHS & P VAlue \\
\hline Demographic features: & $\mathrm{N}=281$ & $\mathrm{~N}=253$ & $\mathrm{~N}=28$ & \\
\hline Age, years & $69.0(62.0-77.0)$ & $69.0(62.0-78.0)$ & $68.0(62.0-74.5)$ & 0.66 \\
\hline \multicolumn{5}{|l|}{ Sex: } \\
\hline -Male & $191(68.0 \%)$ & $172(68.0 \%)$ & $19(67.9 \%)$ & \multirow{2}{*}{1} \\
\hline -Female & $90(32.0 \%)$ & $81(32.0 \%)$ & $9(32.1 \%)$ & \\
\hline Comorbidities: $\dagger$ & $\mathrm{N}=281$ & $\mathrm{~N}=253$ & $\mathrm{~N}=28$ & \\
\hline Hypertension & $109(38.8 \%)$ & $99(39.1 \%)$ & $10(35.7 \%)$ & 0.883 \\
\hline Diabetes & $40(14.2 \%)$ & $35(13.8 \%)$ & $5(17.9 \%)$ & 0.569 \\
\hline CHD & $32(11.4 \%)$ & $29(11.5 \%)$ & $3(10.7 \%)$ & 1.000 \\
\hline Cancer & $23(8.2 \%)$ & $18(7.1 \%)$ & $5(17.9 \%)$ & 0.064 \\
\hline Cerebral infarction & $12(4.3 \%)$ & $11(4.3 \%)$ & $1(3.6 \%)$ & 1.000 \\
\hline Pulmonary tuberculosis & $9(3.2 \%)$ & $8(3.2 \%)$ & $1(3.6 \%)$ & 1.000 \\
\hline Chronic bronchitis & $8(2.8 \%)$ & $8(3.2 \%)$ & $0(0.0 \%)$ & 1.000 \\
\hline COPD & $4(1.4 \%)$ & $4(1.6 \%)$ & $0(0.0 \%)$ & 1.000 \\
\hline Hepatitis and liver cirrhosis & $7(2.5 \%)$ & $6(2.4 \%)$ & $1(3.6 \%)$ & 0.524 \\
\hline Others & $75(26.7 \%)$ & $66(26.1 \%)$ & $9(32.1 \%)$ & 0.644 \\
\hline Initial symptoms: $\dagger$ & $\mathrm{N}=256$ & $\mathrm{~N}=233$ & $\mathrm{~N}=23$ & \\
\hline Fever & $195(76.2 \%)$ & $177(76.0 \%)$ & $18(78.3 \%)$ & 1 \\
\hline Dyspnea & $129(50.4 \%)$ & $118(50.6 \%)$ & $11(47.8 \%)$ & 0.969 \\
\hline Cough & $161(62.9 \%)$ & $154(66.1 \%)$ & $7(30.4 \%)$ & 0.002 \\
\hline Expectoration & $106(41.4 \%)$ & $102(43.8 \%)$ & $4(17.4 \%)$ & 0.026 \\
\hline Diarrhea & $59(23.0 \%)$ & $53(22.7 \%)$ & $6(26.1 \%)$ & 0.918 \\
\hline Fatigue & $51(19.9 \%)$ & $40(17.2 \%)$ & $11(47.8 \%)$ & 0.001 \\
\hline Chest tightness & $41(16.0 \%)$ & $35(15.0 \%)$ & $6(26.1 \%)$ & 0.227 \\
\hline Chills & $25(9.8 \%)$ & $23(9.9 \%)$ & $2(8.7 \%)$ & 1.000 \\
\hline Poor appetite & $21(8.2 \%)$ & $19(8.2 \%)$ & $2(8.7 \%)$ & 1.000 \\
\hline Muscle pain & $15(5.9 \%)$ & $12(5.2 \%)$ & $3(13.0 \%)$ & 0.141 \\
\hline Headache & $13(5.1 \%)$ & $12(5.2 \%)$ & $1(4.3 \%)$ & 1.000 \\
\hline Vertigo & $19(7.4 \%)$ & $18(7.7 \%)$ & $1(4.3 \%)$ & 1.000 \\
\hline Others & $20(7.8 \%)$ & $18(7.7 \%)$ & $2(8.7 \%)$ & 0.697 \\
\hline
\end{tabular}


Table 1. Continued

\begin{tabular}{|c|c|c|c|c|}
\hline INDICATORS & TOTAL & NON-SUDDEN DEATHS & SUDDEN DEATHS & P VALUE \\
\hline CT findings: & $\mathrm{N}=64$ & $\mathrm{~N}=60$ & $\mathrm{~N}=4$ & \\
\hline Ground-glass opacity & $37(57.8 \%)$ & $36(60.0 \%)$ & $1(25.0 \%)$ & 0.302 \\
\hline Patchy shadows & $48(75.0 \%)$ & $44(73.3 \%)$ & $4(100.0 \%)$ & 0.564 \\
\hline Fibrous stripes & $9(14.1 \%)$ & $9(15.0 \%)$ & $0(0.0 \%)$ & 1.000 \\
\hline Pleural thickening & $27(42.2 \%)$ & $25(41.7 \%)$ & $2(50.0 \%)$ & 1.000 \\
\hline Nodules & $6(9.4 \%)$ & $5(8.3 \%)$ & $1(25.0 \%)$ & 0.332 \\
\hline Lymphadenia & $28(43.8 \%)$ & $27(45.0 \%)$ & $1(25.0 \%)$ & 0.625 \\
\hline Bilateral pulmonary & $64(100.0 \%)$ & $60(100.0 \%)$ & $4(100.0 \%)$ & 1.000 \\
\hline Right lung & $6(9.4 \%)$ & $5(8.3 \%)$ & $1(25.0 \%)$ & 0.332 \\
\hline Left lung & $6(9.4 \%)$ & $5(8.3 \%)$ & $1(25.0 \%)$ & 0.332 \\
\hline Treatment: & $\mathrm{N}=281$ & $\mathrm{~N}=253$ & $\mathrm{~N}=28$ & \\
\hline Antiviral therapy & $126(44.8 \%)$ & $113(44.7 \%)$ & $13(46.4 \%)$ & 1 \\
\hline Antibiotic therapy & $272(96.8 \%)$ & $246(97.2 \%)$ & $26(92.9 \%)$ & 0.223 \\
\hline Use of immune globulin & $163(58.0 \%)$ & $155(61.3 \%)$ & $8(28.6 \%)$ & 0.002 \\
\hline Use of corticosteroid & $252(89.7 \%)$ & $230(90.9 \%)$ & $22(78.6 \%)$ & 0.053 \\
\hline Renal-replacement therapy & $102(36.3 \%)$ & $82(32.4 \%)$ & $20(71.4 \%)$ & $<0.001$ \\
\hline \multicolumn{5}{|l|}{ Oxygen support: } \\
\hline -Nasal cannula & $100(35.6 \%)$ & $82(32.4 \%)$ & $18(64.3 \%)$ & 0.002 \\
\hline -Non-invasive ventilation & $74(26.3 \%)$ & $68(26.9 \%)$ & $6(21.4 \%)$ & 0.693 \\
\hline -Invasive ventilation & $105(37.4 \%)$ & $103(40.7 \%)$ & $2(7.1 \%)$ & 0.001 \\
\hline ECOM & $6(2.1 \%)$ & $6(2.4 \%)$ & $0(0.0 \%)$ & 1.000 \\
\hline Admission to ICU & $147(52.3 \%)$ & $143(56.5 \%)$ & $4(14.3 \%)$ & $<0.001$ \\
\hline
\end{tabular}

CHD - coronary heart disease, COPD - chronic obstructive pulmonary disease, ECMO - extracorporeal membrane oxygenation, ICU intensive care unit

*Continuous variables were described as median (IQR). $P$ values were calculated by Wilcoxon rank sum test. Categorical variables were expressed as number (\%). $P$ values were calculated by Pearson $\chi^{2}$ test or Fisher exact test.

†Other comorbidities: cerebrovascular disease, chronic kidney disease, emphysema, hyperuricemia, atrial fibrillation, Parkinson disease, Alzheimer disease, anxiety, hyperthyroidism, hypothyroidism, systemic lupus erythematosus, peptic ulcer; Other initial symptoms: hemoptysis, stomachache, palpitation, anorexia, aversion.

\section{Radiographic features of chest CT scan findings}

Chest CT images were collected from 64 patients. Ground-glass opacity, patchy shadows and pleural thickening were the most common characteristics in both sudden deaths $(1,25.0 \%$; $4,100.0 \%$; and $2,50.0 \%)$ and non-sudden deaths $(36,60.0 \%$; 44, 73.3\%; and 25, 41.7\%). All patients had bilateral pulmonary injury. No significant differences were detected between the two groups. Results are shown in Table 1.

\section{Treatments}

A majority of the patients $(272,96.8 \%)$ received antibiotic therapy, $44.8 \%$ received antiviral therapy, and $89.7 \%$ used corticosteroid. Compared with patients with sudden death, non-sudden deaths had a higher percentage of immune globulin use $(61.3 \%$ vs $28.6 \%, P=0.002)$, while lower percentage of renal-replacement therapy (32.4\% vs $71.4 \%$ ). Invasive mechanical ventilation was initiated in more non-sudden deaths than sudden deaths ( $40.7 \%$ vs $7.1 \%, P=0.001$ ). Extracorporeal membrane oxygenation was performed in 6 patients (2.1\%). 147 patients were admitted to the intensive care unit (ICU), with a higher percentage among non-sudden deaths than sudden deaths (56.5\% vs 14.3\%). Results are shown in Table 1.

\section{Characteristics of laboratory examinations}

For blood routine results, the levels of eosinophils count and the percentage of eosinophils were much lower in sudden deaths than in non-sudden deaths $\left(0.002 \pm 0.006\right.$ vs $0.025 \pm 0.090 \times 10^{9} / \mathrm{L}$ and $0.014 \pm 0.052$ vs $0.238 \pm 0.719 \%)$. The percentage of monocytes was also lower in sudden deaths than in non-sudden deaths (3.30, IQR $=2.58-4.25$ vs $4.90, \mathrm{IQR}=2.80-8.00 \%$ ), but the values were roughly within the normal physiological range. Neutrophils count and the percentage of neutrophils were much higher in sudden deaths than in non-sudden deaths $\left(10.34, \mathrm{IQR}=7.70-15.83\right.$ vs $7.30, \mathrm{IQR}=4.49-11.54 \times 10^{9} / \mathrm{L}$ and $89.15, \mathrm{IQR}=86.88-92.40$ vs $86.70, \mathrm{IQR}=79.10-92.20 \%$ ). In terms of immune cells, the levels of lymphocytes, $\mathrm{CD}^{+} \mathrm{T}$ cells, $\mathrm{CD} 3^{+} \mathrm{CD} 4^{+}$ $\mathrm{T}$ cells, $\mathrm{CD} 3^{+} \mathrm{CD}^{+} \mathrm{T}$ cells, $\mathrm{CD} 3^{-} \mathrm{CD} 16^{+} \mathrm{CD} 56^{+} \mathrm{NK}$ cells in both groups were lower than normal, but the differences between the two groups were not statistically significant.

Inflammatory examination results showed that the concentrations of procalcitonin and C-reactive protein (CRP) were much higher in sudden deaths than in non-sudden deaths $(0.54$, IQR $=0.36-1$. 11 vs 0.23 , IQR $=0.12$ $0.88 \mathrm{ng} / \mathrm{mL}$; and 151.35, IQR=122.40-202.02 vs $100.65, \mathrm{IQR}=57.12-161.07 \mathrm{mg} / \mathrm{L})$. 
In coagulation examinations, sudden deaths showed longer prothrombin time, higher prothrombin time international normalized ratio and lower prothrombin activity when compared with non-sudden deaths (16.30, $\mathrm{IQR}=14.90-20.18$ vs $15.30, \mathrm{IQR}=14.10-16.70$ seconds; $1.29, \mathrm{IQR}=1.16-1.72$ vs $1.20, \mathrm{IQR}=1.09-1.35$; and 67.50, IQR $=47.00-79.00$ vs 76.00, IQR=64.00-87.00\%). Besides, the levels of D-dimer were much higher in sudden deaths than that in non-sudden deaths (7.96, IQR $=2.93-21.00$ vs $3.36, \mathrm{IQR}=1.31-14.16 \mathrm{ug} / \mathrm{mL}$ )

Compared with non-sudden deaths, sudden deaths had higher levels of alanine aminotransferase (39.50, $\mathrm{IQR}=28.50-60.00 \mathrm{vs} 24.00, \mathrm{IQR}=17.00-41.00 \mathrm{U} / \mathrm{L})$, aspartate aminotransferase $(60.00, \mathrm{IQR}=37.75-85.25 \mathrm{vs}$ $40.00, \mathrm{IQR}=28.00-58.00 \mathrm{U} / \mathrm{L})$, gamma-glutamyl transferase (50.50, IQR $=34.75-97.00$ vs 38.00, IQR =24.00$65.00 \mathrm{U} / \mathrm{L})$, lactate dehydrogenase (619.00, IQR $=549.25-884.50$ vs 489.00, IQR=357.00-648.00 U/L), alkaline phosphatase $(93.50, \mathrm{IQR}=69.75-166.00$ vs $76.00, \mathrm{IQR}=59.00-102.00 \mathrm{U} / \mathrm{L})$ and N-terminal pro-brain natriuretic peptide (2044.00, IQR=980.50-6315.00 vs 843.00, IQR=330.00-2401.00 pg/mL). There were no significant differences in other factors of laboratory examinations. Results are shown in Table 2.

Table 2. Laboratory findings of the study patients*

\begin{tabular}{|c|c|c|c|c|c|c|c|c|}
\hline \multirow{2}{*}{$\begin{array}{l}\text { INDICATORS } \\
\text { Inflammatory factors: }\end{array}$} & \multicolumn{2}{|l|}{ NORMAL RANGE } & \multirow[t]{2}{*}{ TOTAL } & \multicolumn{2}{|c|}{ NON-SUDDEN DEATHS } & \multicolumn{2}{|r|}{ SUDDEN DEATHS } & \multirow[t]{2}{*}{ P VALUE } \\
\hline & & & & & & & & \\
\hline Procalcitonin, ng/mL & $<0.05$ & $\mathrm{~N}=261$ & $0.29(0.12-0.89)$ & $\mathrm{N}=240$ & $0.23(0.12-0.88)$ & $N=21$ & $0.54(0.36-1.11)$ & 0.029 \\
\hline CRP, mg/L & $<1.0$ & $\mathrm{~N}=276$ & $105.60(59.33-164.43)$ & $\mathrm{N}=250$ & $100.65(57.12-161.07)$ & $\mathrm{N}=26$ & $151.35(122.40-202.02)$ & 0.001 \\
\hline \multicolumn{9}{|l|}{ Cytokines: } \\
\hline IL-6, pg/mL & $0.0-7.0$ & $\mathrm{~N}=202$ & $61.34(29.27-151.45)$ & $\mathrm{N}=193$ & $59.69(26.56-141.90)$ & $\mathrm{N}=9$ & $146.80(63.03-158.90)$ & 0.145 \\
\hline IL-10, pg/mL & $0.0-9.1$ & $\mathrm{~N}=199$ & $10.30(6.35-18.70)$ & $\mathrm{N}=191$ & $10.40(6.30-18.55)$ & $\mathrm{N}=8$ & $9.25(6.70-23.70)$ & 0.895 \\
\hline IL-8, pg/mL & $0.0-62.0$ & $\mathrm{~N}=200$ & $28.40(16.35-61.82)$ & $\mathrm{N}=192$ & $28.35(16.15-63.52)$ & $\mathrm{N}=8$ & $30.55(20.33-38.88)$ & 0.884 \\
\hline $\mathrm{IL}-1 \beta, \mathrm{pg} / \mathrm{mL}$ & $0.0-5.0$ & $N=200$ & $5.00(5.00-6.93)$ & $N=192$ & $5.00(5.00-7.03)$ & $\mathrm{N}=8$ & $5.00(5.00-5.00)$ & 0.248 \\
\hline $\mathrm{IL}-2 \mathrm{R}, \mathrm{U} / \mathrm{mL}$ & $223.0-710.0$ & $\mathrm{~N}=198$ & $1148.0(740.3-1615.0)$ & $\mathrm{N}=190$ & $1126.0(731.8-1599.3)$ & $\mathrm{N}=8$ & $1340.5(1067-1782.25)$ & 0.346 \\
\hline \multicolumn{9}{|l|}{ Blood routine: } \\
\hline Leucocytes count, $\times 10^{9} / \mathrm{L}$ & $3.50-9.50$ & $\mathrm{~N}=281$ & $8.91(6.00-13.03)$ & $\mathrm{N}=253$ & $8.46(5.73-12.89)$ & $\mathrm{N}=28$ & $11.27(8.68-17.90)$ & 0.011 \\
\hline Erythrocytes count, $\times 10^{12} \mathrm{~L}$ & $4.30-5.80$ & $\mathrm{~N}=281$ & $4.17(3.60-4.63)$ & $\mathrm{N}=253$ & $4.17(3.61-4.62)$ & $\mathrm{N}=28$ & $4.19(3.52-4.70)$ & 0.822 \\
\hline Monocytes, \% & $3.0-10.0$ & $\mathrm{~N}=281$ & $4.60(2.70-7.50)$ & $\mathrm{N}=253$ & $4.90(2.80-8.00)$ & $\mathrm{N}=28$ & $3.30(2.58-4.25)$ & 0.003 \\
\hline Monocytes count, $\times 10^{9} / \mathrm{L}$ & $0.10-0.60$ & $N=281$ & $0.40(0.26-0.62)$ & $\mathrm{N}=253$ & $0.40(0.27-0.63)$ & $N=28$ & $0.42(0.24-0.54)$ & 0.753 \\
\hline Neutrophils, \% & $40.0-75.0$ & $\mathrm{~N}=281$ & $87.10(79.90-92.20)$ & $\mathrm{N}=253$ & $86.70(79.10-92.20)$ & $N=28$ & $89.15(86.88-92.40)$ & 0.036 \\
\hline Neutrophils count, $\times 10^{9} / \mathrm{L}$ & $1.80-6.30$ & $\mathrm{~N}=281$ & $7.75(4.53-11.63)$ & $\mathrm{N}=253$ & $7.30(4.49-11.54)$ & $N=28$ & $10.34(7.70-15.83)$ & 0.006 \\
\hline \multirow{2}{*}{ Eosinophils, \% } & \multirow{2}{*}{$0.4-8.0$} & $\mathrm{~N}=281$ & $0.00(0.00-0.10)$ & $\mathrm{N}=253$ & $0.00(0.00-0.10)$ & $N=28$ & $0.00(0.00-0.00)$ & 0.004 \\
\hline & & $\mathrm{N}=281$ & $0.215 \pm 0.686$ & $\mathrm{~N}=253$ & $0.238 \pm 0.719$ & $\mathrm{~N}=28$ & $0.014 \pm 0.052$ & \\
\hline \multirow{2}{*}{ Eosinophils count, $\times 10^{9} / \mathrm{L}$} & \multirow{2}{*}{$0.02-0.52$} & $\mathrm{~N}=281$ & $0.00(0.00-0.01)$ & $\mathrm{N}=253$ & $0.00(0.00-0.01)$ & $\mathrm{N}=28$ & $0.00(0.00-0.00)$ & 0.006 \\
\hline & & $\mathrm{N}=281$ & $0.023 \pm 0.086$ & $\mathrm{~N}=253$ & $0.025 \pm 0.090$ & $\mathrm{~N}=28$ & $0.002 \pm 0.006$ & \\
\hline Basophils, \% & $0.0-1.0$ & $\mathrm{~N}=281$ & $0.10(0.00-0.20)$ & $\mathrm{N}=253$ & $0.10(0.00-0.20)$ & $N=28$ & $0.10(0.08-0.12)$ & 0.545 \\
\hline Basophils count, $\times 10^{9} / \mathrm{L}$ & $0.00-0.10$ & $\mathrm{~N}=281$ & $0.01(0.00-0.02)$ & $\mathrm{N}=253$ & $0.01(0.00-0.02)$ & $\mathrm{N}=28$ & $0.01(0.01-0.02)$ & 0.423 \\
\hline Hemoglobin, $\mathrm{g} / \mathrm{L}$ & $130.0-175.0$ & $N=281$ & $128.00(112.00-143.00)$ & $\mathrm{N}=253$ & $128.00(111.00-143.00)$ & $\mathrm{N}=28$ & $122.00(112.75-142.25)$ & 0.761 \\
\hline Platelets count, $\times 10^{9} / \mathrm{L}$ & $125.0-350.0$ & $N=281$ & $159.00(111.00-224.00)$ & $\mathrm{N}=253$ & $161.00(112.00-224.00)$ & $\mathrm{N}=28$ & $147.00(87.75-223.50)$ & 0.580 \\
\hline \multicolumn{9}{|l|}{ Lymphocytes: } \\
\hline Lymphocytes, \% & $20.0-50.0$ & $\mathrm{~N}=281$ & $7.10(4.10-12.20)$ & $\mathrm{N}=253$ & $7.20(4.10-12.60)$ & $\mathrm{N}=28$ & $6.90(4.33-9.12)$ & 0.289 \\
\hline Lymphocytes count, $\times 10^{9} / \mathrm{L}$ & $1.10-3.20$ & $\mathrm{~N}=281$ & $0.63(0.44-0.85)$ & $\mathrm{N}=253$ & $0.61(0.43-0.84)$ & $\mathrm{N}=28$ & $0.66(0.51-0.90)$ & 0.230 \\
\hline $\mathrm{CD}^{+} \mathrm{T}$ cell count $/ \mu \mathrm{L}$ & $690-2540$ & $\mathrm{~N}=54$ & $276.50(132.75-408.50)$ & $\mathrm{N}=49$ & $269.00(130.00-404.00)$ & $\mathrm{N}=5$ & $379.00(332.00-448.00)$ & 0.395 \\
\hline $\mathrm{CD}^{+}{ }^{+} \mathrm{CD} 4^{+} \mathrm{T}$ cell count $/ \mu \mathrm{L}$ & $410-1590$ & $\mathrm{~N}=54$ & $170.50(93.25-257.50)$ & $\mathrm{N}=49$ & $166.00(93.00-243.00)$ & $\mathrm{N}=5$ & $264.00(253.00-275.00)$ & 0.257 \\
\hline $\mathrm{CD}^{+}{ }^{+} \mathrm{CD}^{+} \mathrm{T}$ cell count $/ \mu \mathrm{L}$ & $190-1140$ & $\mathrm{~N}=54$ & $62.00(29.25-127.00)$ & $\mathrm{N}=49$ & $61.00(29.00-124.00)$ & $\mathrm{N}=5$ & $110.00(56.00-158.00)$ & 0.447 \\
\hline $\mathrm{CD}^{+} / \mathrm{CD}^{+} \mathrm{T}$ cell count$/ \mathrm{\mu L}$ & $1.02-1.94$ & $\mathrm{~N}=54$ & $2.86(1.82-4.72)$ & $\mathrm{N}=49$ & $2.97(1.83-4.79)$ & $\mathrm{N}=5$ & $2.40(1.79-3.95)$ & 0.633 \\
\hline $\begin{array}{l}\text { CD3-CD16+CD56 } 6^{+} \text {NK cell } \\
\text { count } / \mu \mathrm{L}\end{array}$ & $88-64$ & $\mathrm{~N}=54$ & $36.50(16.00-74.75)$ & $\mathrm{N}=49$ & $35.00(16.00-74.00)$ & $N=5$ & $43.00(26.00-114.00)$ & 0.665 \\
\hline CD19+ B cell count $/ \mu \mathrm{L}$ & $77-736$ & $\mathrm{~N}=54$ & $73.50(40.25-143.00)$ & $\mathrm{N}=49$ & $69.00(40.00-130.00)$ & $N=5$ & $168.00(75.00-229.00)$ & 0.257 \\
\hline \multicolumn{9}{|l|}{ Coagulation profiles: } \\
\hline APTT, s & $26.0-42.0$ & $\mathrm{~N}=250$ & $40.25(36.23-46)$ & $\mathrm{N}=229$ & $40.10(36.10-46.00)$ & $\mathrm{N}=21$ & $42.10(38.00-48.30)$ & 0.309 \\
\hline PT, s & $10.0-15.0$ & $\mathrm{~N}=281$ & $15.30(14.30-16.90)$ & $\mathrm{N}=253$ & $15.30(14.10-16.70)$ & $\mathrm{N}=28$ & $16.30(14.90-20.18)$ & 0.016 \\
\hline PT-INR & $0.8-1.2$ & $\mathrm{~N}=281$ & $1.20(1.10-1.37)$ & $\mathrm{N}=253$ & $1.20(1.09-1.35)$ & $\mathrm{N}=28$ & $1.29(1.16-1.72)$ & 0.017 \\
\hline D-dimer, ug/mL & $<0.5$ & $\mathrm{~N}=275$ & $4.10(1.39-15.41)$ & $\mathrm{N}=247$ & $3.36(1.31-14.16)$ & $\mathrm{N}=28$ & $7.96(2.93-21.00)$ & 0.004 \\
\hline Fibrinogen, g/L & $2.0-4.0$ & $\mathrm{~N}=248$ & $4.85 \pm 2.08$ & $\mathrm{~N}=227$ & $4.88 \pm 2.08$ & $\mathrm{~N}=21$ & $4.57 \pm 2.18$ & 0.510 \\
\hline PTA, $\%$ & $70.0-140.0$ & $\mathrm{~N}=281$ & $75.00(62.00-86.00)$ & $\mathrm{N}=253$ & $76.00(64.00-87.00)$ & $\mathrm{N}=28$ & $67.50(47.00-79.00)$ & 0.019 \\
\hline TT, s & $11.0-17.0$ & $\mathrm{~N}=248$ & $17.00(15.80-19.10)$ & $N=228$ & $17.10(15.88-19.10)$ & $N=20$ & $16.45(15.75-18.38)$ & 0.545 \\
\hline \multicolumn{9}{|l|}{ Electrolytes: } \\
\hline Potassium, mmol/L & $3.50-5.30$ & $\mathrm{~N}=281$ & $4.33(3.89-4.84)$ & $\mathrm{N}=253$ & $4.33(3.87-4.84)$ & $N=28$ & $4.29(3.92-4.85)$ & 0.890 \\
\hline Sodium, $\mathrm{mmol} / \mathrm{L}$ & $137.00-147.00$ & $\mathrm{~N}=281$ & $138.80(135.40-142.70)$ & $\mathrm{N}=253$ & $138.50(134.80-142.40)$ & $\mathrm{N}=28$ & $141.25(137.10-144.40)$ & 0.054 \\
\hline
\end{tabular}


Table 2. Continued

\begin{tabular}{|c|c|c|c|c|c|c|c|c|}
\hline INDICATORS & NORMAL RANGE & & TOTAL & & N-SUDDEN DEATHS & & SUDDEN DEATHS & P VALUE \\
\hline Chloridion, mmol/L & $90.00-110.00$ & $\mathrm{~N}=281$ & $100.20(96.20-104.30)$ & $N=253$ & $100.00(96.20-104.00)$ & $\mathrm{N}=28$ & $102.00(97.17-106.10)$ & 0.210 \\
\hline Calcium, $\mathrm{mmol} / \mathrm{L}$ & $2.11-2.52$ & $\mathrm{~N}=281$ & $2.06(1.99-2.14)$ & $\mathrm{N}=253$ & $2.06(1.99-2.15)$ & $\mathrm{N}=28$ & $2.04(1.97-2.09)$ & 0.196 \\
\hline $\mathrm{PHOS}, \mathrm{mmol} / \mathrm{L}$ & $0.85-1.51$ & $\mathrm{~N}=194$ & $0.96(0.75-1.28)$ & $\mathrm{N}=189$ & $0.96(0.75-1.28)$ & $\mathrm{N}=5$ & $0.89(0.77-1.43)$ & 0.799 \\
\hline Magnesium, mmol/L & $0.73-1.06$ & $\mathrm{~N}=195$ & $0.91(0.82-1.02)$ & $\mathrm{N}=190$ & $0.90(0.82-1.01)$ & $\mathrm{N}=5$ & $0.93(0.87-1.03)$ & 0.490 \\
\hline Organ damage index: & & & & & & & & \\
\hline ALT, U/L & $\leq 41$ & $\mathrm{~N}=281$ & $27.00(18.00-42.00)$ & $\mathrm{N}=253$ & $24.00(17.00-41.00)$ & $\mathrm{N}=28$ & $39.50(28.50-60.00)$ & 0.001 \\
\hline AST, U/L & $\leq 40$ & $\mathrm{~N}=281$ & $41.00(29.00-59.00)$ & $\mathrm{N}=253$ & $40.00(28.00-58.00)$ & $\mathrm{N}=28$ & $60.00(37.75-85.25)$ & 0.003 \\
\hline GGT, U/L & $6-42$ & $\mathrm{~N}=281$ & $39.00(25.00-69.00)$ & $\mathrm{N}=253$ & $38.00(24.00-65.00)$ & $\mathrm{N}=28$ & $50.50(34.75-97.00)$ & 0.031 \\
\hline Total bilirubin, $\mu \mathrm{mol} / \mathrm{L}$ & $\leq 26$ & $\mathrm{~N}=281$ & $12.30(9.00-18.70)$ & $N=253$ & $12.30(9.00-18.70)$ & $\mathrm{N}=28$ & $12.00(8.75-18.18)$ & 0.917 \\
\hline Direct bilirubin, $\mu \mathrm{mol} / \mathrm{L}$ & $\leq 8.0$ & $\mathrm{~N}=280$ & $6.15(4.40-9.60)$ & $\mathrm{N}=252$ & $6.10(4.40-9.30)$ & $\mathrm{N}=28$ & $6.30(4.42-10.40)$ & 0.630 \\
\hline Indirect bilirubin, $\mu \mathrm{mol} / \mathrm{L}$ & $\leq 16.8$ & $\mathrm{~N}=278$ & $5.95(4.10-8.40)$ & $\mathrm{N}=250$ & $5.95(4.23-8.40)$ & $\mathrm{N}=28$ & $5.90(3.53-8.25)$ & 0.747 \\
\hline ALB, $g / L$ & $35-52$ & $\mathrm{~N}=280$ & $31.10(28.00-34.20)$ & $\mathrm{N}=252$ & $31.30(27.98-34.32)$ & $\mathrm{N}=28$ & $29.75(28.17-31.72)$ & 0.157 \\
\hline GLO, g/L & $20-35$ & $\mathrm{~N}=280$ & $35.60(31.48-39.05)$ & $\mathrm{N}=252$ & $35.65(31.30-39.05)$ & $\mathrm{N}=28$ & $35.40(33.40-39.10)$ & 0.571 \\
\hline Total protein, g/L & 64-83 & $\mathrm{N}=280$ & $66.50(61.82-70.82)$ & $\mathrm{N}=252$ & $66.45(61.60-71.00)$ & $\mathrm{N}=28$ & $66.60(62.50-68.80)$ & 0.550 \\
\hline ALB/GLO & $1.50-2.50$ & $\mathrm{~N}=280$ & $0.86(0.75-1.00)$ & $\mathrm{N}=252$ & $0.87(0.76-1.01)$ & $N=28$ & $0.84(0.74-0.92)$ & 0.305 \\
\hline Total bile acid, $\mu \mathrm{mol} / \mathrm{L}$ & $\leq 10$ & $\mathrm{~N}=177$ & $3.40(1.70-6.50)$ & $\mathrm{N}=173$ & $3.40(1.70-6.50)$ & $\mathrm{N}=4$ & $3.35(1.38-6.12)$ & 0.726 \\
\hline Creatinine, $\mu \mathrm{mol} / \mathrm{L}$ & $45-84$ & $\mathrm{~N}=281$ & $87.00(67.00-114.00)$ & $\mathrm{N}=253$ & $86.00(67.00-113.00)$ & $\mathrm{N}=28$ & $95.50(64.75-119.00)$ & 0.760 \\
\hline Urea, $\mathrm{mmol} / \mathrm{L}$ & $3.1-8.8$ & $\mathrm{~N}=279$ & $8.30(5.60-12.90)$ & $\mathrm{N}=251$ & $8.20(5.60-12.80)$ & $\mathrm{N}=28$ & $9.74(5.68-13.33)$ & 0.405 \\
\hline Uric acid, $\mu \mathrm{mol} / \mathrm{L}$ & $142.8-339.2$ & $\mathrm{~N}=279$ & $265.20(194.00-371.50)$ & $\mathrm{N}=251$ & $265.20(189.50-373.50)$ & $N=28$ & $291.00(222.75-335.25)$ & 0.421 \\
\hline Total cholesterol, $\mathrm{mmol} / \mathrm{L}$ & $<5.18$ & $\mathrm{~N}=279$ & $3.33(2.83-3.96)$ & $\mathrm{N}=251$ & $3.33(2.83-3.96)$ & $\mathrm{N}=28$ & $3.25(2.82-3.69)$ & 0.633 \\
\hline Triglyceride, $\mathrm{mmol} / \mathrm{L}$ & $0.56-1.7$ & $\mathrm{~N}=178$ & $1.54(1.18-2.19)$ & $\mathrm{N}=171$ & $1.53(1.18-2.17)$ & $\mathrm{N}=7$ & $1.69(1.12-2.19)$ & 0.991 \\
\hline $\mathrm{HDL}-\mathrm{C}, \mathrm{mmol} / \mathrm{L}$ & $0.82-1.96$ & $\mathrm{~N}=177$ & $0.78 \pm 0.25$ & $\mathrm{~N}=170$ & $0.78 \pm 0.25$ & $\mathrm{~N}=7$ & $0.76 \pm 0.32$ & 0.854 \\
\hline LDL-C, mmol/L & $0.8-3.36$ & $\mathrm{~N}=176$ & $1.90(1.43-2.53)$ & $\mathrm{N}=169$ & $1.92(1.43-2.54)$ & $\mathrm{N}=7$ & $1.86(1.56-2.28)$ & 0.797 \\
\hline Glucose, $\mathrm{mmol} / \mathrm{L}$ & $3.89-6.11$ & $\mathrm{~N}=275$ & $7.84(6.37-11.06)$ & $\mathrm{N}=249$ & $7.71(6.32-10.95)$ & $N=26$ & $8.95(7.30-13.76)$ & 0.067 \\
\hline $\mathrm{LDH}, \mathrm{U} / \mathrm{L}$ & $135-225$ & $\mathrm{~N}=277$ & $504.00(364.00-669.00)$ & $\mathrm{N}=251$ & $489.00(357.00-648.00)$ & $\mathrm{N}=26$ & $619.00(549.25-884.50)$ & 0.001 \\
\hline Alkaline phosphatase, U/L & $35-105$ & $\mathrm{~N}=281$ & $78.00(60.00-107.00)$ & $\mathrm{N}=253$ & $76.00(59.00-102.00)$ & $\mathrm{N}=28$ & $93.50(69.75-166.00)$ & 0.007 \\
\hline Choline esterase, U/L & $4000-12000$ & $\mathrm{~N}=181$ & $4947.48 \pm 1618.62$ & $\mathrm{~N}=177$ & $4948.79 \pm 1616.65$ & $\mathrm{~N}=4$ & $4889.50 \pm 1965.47$ & 0.942 \\
\hline NT-proBNP, pg/mL & $<350$ & $\mathrm{~N}=252$ & $888.50(362.75-2566.50)$ & $\mathrm{N}=233$ & $843.00(330.00-2401.00)$ & $\mathrm{N}=19$ & $2044.00(980.50-6315.00)$ & 0.008 \\
\hline Myoglobin, $\mathrm{ng} / \mathrm{mL}$ & $10-80$ & $\mathrm{~N}=178$ & $237.20(100.68-1200.00)$ & $\mathrm{N}=170$ & $237.20(100.68-1200.00)$ & $\mathrm{N}=8$ & $258.10(97.18-1150.88)$ & 0.952 \\
\hline hs-cTnI, pg/mL & $<0.1$ & $\mathrm{~N}=261$ & $35.30(11.20-194.70)$ & $\mathrm{N}=239$ & $31.10(10.90-192.90)$ & $N=22$ & $65.00(24.17-246.02)$ & 0.082 \\
\hline Creatine kinase, U/L & $30-170$ & $\mathrm{~N}=192$ & $129.00(69.75-332.75)$ & $\mathrm{N}=184$ & $127.50(68.75-328.75)$ & $\mathrm{N}=8$ & $290.00(107.50-788.25)$ & 0.153 \\
\hline
\end{tabular}

Furthermore, logistic models were employed to explore risk factors from laboratory examinations associated with the sudden death of COVID-19 patients. Both in univariate and multivariate logistic regression models, $\mathrm{CRP}(\mathrm{OR}=1.01, P=0.003)$, percentage of monocytes ( $\mathrm{OR}=0.78, P=0.004)$, percentage of neutrophils $(\mathrm{OR}=1.07, \mathrm{P}=0.023)$, neutrophils count $(\mathrm{OR}=1.09, \mathrm{P}=0.010)$, $\mathrm{D}$-dimer $(\mathrm{OR}=1.06, \mathrm{P}=0.011)$, prothrombin activity $(\mathrm{OR}=0.98, P=0.020)$, gamma-glutamyl transferase $(\mathrm{OR}=1.00,95 \% \mathrm{CI} 1.00-1.01, P=0.034)$ and alkaline phosphatase $(\mathrm{OR}=1.01, P<0.001)$ were identified as being associated with sudden death of COVID-19 patients. Results are shown in Table 3.

\section{DISCUSSION}

We report here a total of 281 deceased patients with COVID-19, and comprehensively described the major differences in clinical characteristics between sudden deaths and non-sudden deaths. The median age of deceased patients was 69.0 years. Compared with female, male sex was more predominant in deceased patients. Comorbidities were present in more than half of the deceased patients, with hypertension being the most common type, followed by diabetes and coronary heart disease. However, significant differences in age, sex and comorbidities between sudden deaths and non-sudden deaths were not observed, suggesting that although these factors are associated with death in patients with COVID-19 [5,8-10], they are not the cause of rapid deterioration and death in a short period of time. The incidence of symptoms including fever, dyspnea and diarrhea showed no significant differences between sudden deaths and non-sudden deaths. Fatigue was more common in sudden deaths, whereas dry cough and expectoration were more common in non-sudden deaths. Certain symptoms may be helpful for identifying the patients at risk of an acute death. 
Table 3. Factors of laboratory examinations associated with the sudden death of COVID-19 patients

\begin{tabular}{|c|c|c|c|c|}
\hline \multirow{2}{*}{ INDICATORS } & \multicolumn{2}{|c|}{ UNIVARIATE LOGISTIC REGRESSION } & \multicolumn{2}{|c|}{ MULTIVARIATE LOGISTIC REGRESSION * } \\
\hline & OR $(95 \% \mathrm{CI})$ & $P$ value & OR $(95 \%$ CI) & $P$ value \\
\hline \multicolumn{5}{|l|}{ Inflammatory factors: } \\
\hline CRP, mg/L & $1.01(1.00-1.01)$ & 0.003 & $1.01(1.00-1.01)$ & 0.003 \\
\hline \multicolumn{5}{|l|}{ Blood routine: } \\
\hline Monocytes, \% & $0.78(0.66-0.93)$ & 0.004 & $0.76(0.63-0.92)$ & 0.004 \\
\hline Neutrophils, \% & $1.07(1.01-1.13)$ & 0.023 & $1.08(1.02-1.15)$ & 0.015 \\
\hline Neutrophils count, $\times 10^{9} / \mathrm{L}$ & $1.09(1.02-1.16)$ & 0.01 & $1.10(1.03-1.17)$ & 0.007 \\
\hline \multicolumn{5}{|l|}{ Coagulation profiles: } \\
\hline D-dimer, ug/mL & $1.06(1.01-1.11)$ & 0.011 & $1.06(1.01-1.11)$ & 0.014 \\
\hline PTA, \% & $0.98(0.96-1.00)$ & 0.02 & $0.98(0.96-1.00)$ & 0.032 \\
\hline \multicolumn{5}{|l|}{ Organ damage index: } \\
\hline GGT, U/L & $1.00(1.00-1.01)$ & 0.034 & $1.00(1.00-1.01)$ & 0.028 \\
\hline Alkaline phosphatase, U/L & $1.01(1.01-1.02)$ & $<0.001$ & $1.01(1.01-1.02)$ & $<0.001$ \\
\hline
\end{tabular}

The vast majority of deceased patients showed bilateral pulmonary injury and patchy shadows was the most common finding on chest CT images on admission, but the radiographic findings did not differ between sudden deaths and non-sudden deaths. This indicates that chest CT scan is of little significance for predicting sudden death in critical period, and more meaningful laboratory indicators should be included. Deceased patients developed leukocytosis and lymphopenia, suggesting a possible secondary bacterial infection and cellular immune deficiency. It is worth noting that the decrease of eosinophils was more serious in sudden deaths, which is consistent with previous report that eosinophils may predict the outcome of COVID-19 progression [11]. Eosinophils could be an effective marker for monitoring the severity of COVID-19. We also reported that patients with high percentage of neutrophils or neutrophils count had an increased risk of sudden death, probably due to cytokine storm activated by neutrophils [8]. Higher serum levels of inflammatory marker, CRP, was identified as risk factors of sudden death with COVID-19 patients. This marker is important in immunity and immunopathology during virus infection [12-15]. Notably, significantly higher concentrations of alanine aminotransferase, aspartate aminotransferase, gamma-glutamyl transferase, lactate dehydrogenase, alkaline phosphatase and N-terminal pro-brain natriuretic peptide were observed in sudden deaths than in non-sudden deaths, indicating more impaired liver and heart function. In addition, compared with non-sudden deaths, sudden deaths had more severe changes in coagulation profiles, which also suggested more serious liver injury. These results indicated that the rapidly damaged liver and heart function was likely to cause sudden death of patients with COVID-19.

In this study, we could not prove that antiviral treatment was effective. Supportive therapy that protects important organs and eases the symptoms, like oxygen support, especially the comprehensive treatment and continuous care in the ICU, seemed be beneficial. We observed higher percentages of renal-replacement therapy and nasal cannula in sudden deaths than in non-sudden deaths, but we cannot conclude these treatments are futile to COVID-19 patients, as they were in severe condition when they received these treatments.

Since existing evidence is limited, our study of 281 deceased patients with COVID-19 represents the features of deaths and provides relevant clues to find the associations between sudden death of COVID-19 patients and potential risk factors. Additionally, the statistical analyses such as multivariable logistic regression models could adjust for confounders and minimize the bias. Also, several limitations should be considered. First, it was a retrospective, single-center study, not all radiographic or laboratory examinations were performed in all patients. Interpretation of our findings might be limited by the sample size. Larger cohort with more complete standardized data would make more sense. Second, patients were sometimes admitted to hospital late in illness. Data collected for each patient might be from different disease stages, which might lead to bias in clinical characteristics. Symptoms and comorbidities were self-reported by the patients as well as their family members, which might cause reporting bias. Third, patients with COVID-19 might have bacterial or other viral co-infection, which could affect the results of immune response. Forth, as it was a descriptive study, further mechanistic explanation still needs to be clarified. Despite that, our study demonstrated novel information about the characteristics of COVID-19 patients at risk of sudden death. This would help physicians to effectively identify patients with particularly poor prognosis on admission, and give necessary treatment in time, which may ultimately help to reduce the fatality rate. 
Acknowledgments: This study was approved by the Ethics Committee of Tongji Hospital, Tongji Medical College, Huazhong University of Science and Technology. We acknowledge all patients and their families involved in this study and all health-care workers who are fighting against COVID-19.

Funding: This work was supported by SARS-CoV-2 Pneumonia Emergency Technology Public Relations Project of Tongji Medical College, Huazhong University of Science and Technology (2020kfyXGYJ043) and Science and Technology Support Program of Guizhou province ([2020]4Y165, [2020]4Y171).

Authorship contributions: HZ conceived the study, was responsible for the study design and supervised the entire study. FZ, MJ, JS and PP recruited participants and collected data. JT, YW, NY, KT, MJ, XZ, XW and SN performed data analysis. NY, JT, KT, MJ, and XZ wrote the manuscript. XS, SN, JS, KH and KL performed literature search, contributed to formatting the manuscript. All authors participated in data interpretation, and revised the manuscript. In addition, all authors agree to be accountable for all aspects of this study to ensure that questions related to the accuracy or integrity of any part of the work are properly investigated and resolved.

Competing interests: The authors completed the ICMJE Unified Competing Interest form (available upon request from the corresponding author), and declare no conflicts of interest.

1 Huang C, Wang Y, Li X, Ren L, Zhao J, Hu Y, et al. Clinical features of patients infected with 2019 novel coronavirus in Wuhan, China. Lancet. 2020;395:497-506. Medline:31986264 doi:10.1016/S0140-6736(20)30183-5

2 Lu R, Zhao X, Li J, Niu P, Yang B, Wu H, et al. Genomic characterisation and epidemiology of 2019 novel coronavirus: implications for virus origins and receptor binding. Lancet. 2020;395:565-74. Medline:32007145 doi:10.1016/S0140-6736(20)30251-8

3 Ahmad A, Mueller C, Tsamakis K. Covid-19 pandemic: a public and global mental health opportunity for social transformation? BMJ. 2020;369:m1383. Medline:32253252 doi:10.1136/bmj.m1383

4 Miller DG, Pierson L, Doernberg S. The Role of Medical Students During the COVID-19 Pandemic. Ann Intern Med. 2020;173:1456. Medline:32259194 doi:10.7326/M20-1281

5 Jordan RE, Adab P, Cheng KK. Covid-19: risk factors for severe disease and death. BMJ. 2020;368:m1198. Medline:32217618 doi:10.1136/bmj.ml198

6 World Health Organization. Report of the WHO-China Joint Mission on Coronavirus Disease 2019 (COVID-19). Available: https://www.who.int/docs/default-source/coronaviruse/who-china-joint-mission-on-covid-19-final-report. Accessed: 4 March 2020.

7 World Health Organization. Clinical management of severe acute respiratory infection (SARI) when COVID-19 disease is suspected: Interim guidance. Available: https://www.who.int/docs/default-source/coronaviruse/clinical-management-of-novel-cov. Accessed: 2 April 2020.

8 Wu C, Chen X, Ca Y, Xia JA, Zhou X, Xu S, et al. Risk Factors Associated With Acute Respiratory Distress Syndrome and Death in Patients With Coronavirus Disease 2019 Pneumonia in Wuhan, China. JAMA Intern Med. 2020;180:934-43. Medline:32167524 doi:10.1001/jamainternmed.2020.0994

9 Chen T, Wu D, Chen H, Yan W, Yang D, Chen G, et al. Clinical characteristics of 113 deceased patients with coronavirus disease 2019: retrospective study. BMJ. 2020;368:m1091. Medline:32217556 doi:10.1136/bmj.m1091

10 Zhou F, Yu T, Du R, Fan G, Liu Y, Liu Z, et al. Clinical course and risk factors for mortality of adult inpatients with COVID-19 in Wuhan, China: a retrospective cohort study. Lancet. 2020;395:1054-62. Medline:32171076 doi:10.1016/S0140-6736(20)30566-3

11 Liu F, Xu A, Zhang Y, Xuan W, Yan T, Pan K, et al. Patients of COVID-19 may benefit from sustained lopinavir-combined regimen and the increase of eosinophil may predict the outcome of COVID-19 progression. Int J Infect Dis. 2020;95:183-91. Medline:32173576 doi:10.1016/j.ijid.2020.03.013

12 Ingram PR, Inglis T, Moxon D, Speers D. Procalcitonin and C-reactive protein in severe $2009 \mathrm{H} 1 \mathrm{N1}$ influenza infection. Intensive Care Med. 2010;36:528-32. Medline:20069274 doi:10.1007/s00134-009-1746-3

13 Law HK, Cheung CY, Ng HY, Sia SF, Chan YO, Luk W, et al. Chemokine up-regulation in SARS-coronavirus-infected, monocyte-derived human dendritic cells. Blood. 2005;106:2366-74. Medline:15860669 doi:10.1182/blood-2004-10-4166

14 Jiang Y, Xu J, Zhou C, Wu Z, Zhong S, Liu J, et al. Characterization of cytokine/chemokine profiles of severe acute respiratory syndrome. Am J Respir Crit Care Med. 2005;171:850-7. Medline:15657466 doi:10.1164/rccm.200407-857OC

15 Russell CD, Unger SA, Walton M, Schwarze J. The Human Immune Response to Respiratory Syncytial Virus Infection. Clin Microbiol Rev. 2017;30:481-502. Medline:28179378 doi:10.1128/CMR.00090-16 